\title{
The Improvement of Gynecological Gasless Laparoscopic Surgery Using a Subcutaneous Abdominal Wall Lifting Method
}

\section{Ito $\mathbf{H}$, Watanabe $\mathbf{R}$ and Isaka $\mathrm{K}^{*}$}

Department of Obstetrics and Gynecology, Tokyo

Medical University Hospital, Graduate School of Health Innovation, Kanagawa University of Human Services, Japan

*Corresponding author: Keiichi Isaka, Department of Obstetrics and Gynecology, Tokyo Medical University Hospital, 6-7-1, Nishishinjuku, Shinjuku-ku, Tokyo 1600023, J apan

Received: May 29, 2021; Accepted: J une 16, 2021; Published: J une 23, 2021

\begin{abstract}
Background: To evaluate the usefulness of gasless laparoscopic surgery using a Subcutaneous Abdominal Wall Lifting method (SAWL) for gynecological tumors.

Methods: 5309 patients underwent gasless surgery with SAWL in our hospital between April 1993 and December 2015. Patient background as well as the number of annual cases, operation time, estimated blood loss, number of ports, blood transfusion, and shift to laparotomy were examined. With regards to Laparoscopic Cystectomy (LC), Laparoscopic Myomectomy (LM) and Laparoscopic Tubectomy (LT), we divided their cases into two stages-the first stage (FS) that we performed surgery with double-operated ports (1993-2005), and the second stage (SS) after a single-operated port surgery introduction (2008-2015) for comparison.
\end{abstract}

Results: LC was the most frequently performed (2068 patients), followed by LM (1738 patients) and LT (510 patients). A single-operated port laparoscopic surgery, which we called a Gasless Reduced Port Surgery (GRPS), was introduced in 2005, and by 2008 it had accounted for almost $90 \%$ of gasless laparoscopic surgeries overall except for total laparoscopic hysterectomy. The mean operation time was significantly shorter in SS than FS for LC and LT, and it was no significant difference between two stages for LM. The estimated blood loss significantly decreased in SS compared to FS for LM and LT, and no significant difference for LC. The conversion rate in SS was $0.07 \%$.

Conclusion: GRPS is an operative procedure that is superior to the rate of conversion to laparotomy and is aesthetically superior in addition to having advantages of the conventional gasless method.

Keywords: Gynecologic laparoscopic surgery; Subcutaneous abdominal wall lifting method; Gasless surgery; Reduced port surgery

\section{Introduction}

The abdominal wall lifting method can be broadly divided into the Subcutaneous Abdominal Wall Lifting method (SAWL) and the Full-Layer Abdominal Wall Lifting method (FAWL). However, unlike the conventional pneumoperitoneum method by gas insufflation used to ensure the operative field, the abdominal wall is lifted, thereby creating an operative field space in the abdominal cavity. Because gas is not used, it may also be referred to as the gasless method. Among these procedures, SAWL ensures the operative field in the abdominal cavity by lifting the abdominal wall with a steel wire inserted subcutaneously as a support, and was first reported in 1991 by Nagai et al. [1] and Hashimoto et al. [2] of the department of surgery as a novel substitute to the pneumoperitoneum method when performing laparoscopic cholecystectomy. On other hand, FAWL was reported by Gazayerli [3], and Mouret in 1991. However, as both reports indicated that pneumoperitoneum was used in combination, these methods could not be called true abdominal wall lifting. FAWL without pneumoperitoneum include a method using a U-shaped retractor reported by Kitano et al. in 1992 [4] and a method reported by Newman et al. in 1993 [5] involving a lifting instrument that was subsequently commercially released as the Laparolift. However, sales of the Laparolift have currently been discontinued.

From our experience, SAWL is more suitable for gynecology than
FAWL because it does not damage the intestinal tract when the lifting device is inserted and the abdominal wall can be freely moved up and down when removing the ovarian cyst.

In 1993, we introduced SAWL in gynecologic laparoscopic surgery [6]. We believe that this was the first laparoscopic surgery using SAWL worldwide in the field of gynecology

It is said that gasless surgery is an excellent method for safety (rapid suture ligation, stable operation area), operability (early learning ability) and economic efficiency (do not use disposable products) $[2,6]$. On the other hand, problems such as a narrow surgical field, large wounds, cosmetic aspects, and difficulty in dealing with obesity are also pointed out. In order to overcome these disadvantages, we have improved the method for many years and tried to establish a highly useful surgical procedure.

In the present article, we report on the usefulness of gasless surgery using SAWL for gynecological diseases based on our 23 years of experience.

\section{Materials and Methods}

\section{Subjects}

The study included 5309patients who, after providing full informed consent, underwent gasless laparoscopic surgery with SAWL from April 1993 to December 2015 at the Department of 
Obstetrics and Gynecology at Tokyo Medical University Hospital. We received the IRB approval of this university's morals and ethics committee for this study (approval number: SH3628).

Diseases for which conventional laparoscopic surgery by the pneumoperitoneum method is indicated were all eligible for gasless laparoscopic surgery with SAWL.

\section{Instruments and surrounding equipment required for SAWL}

\section{Lifting instrument (Figure 1):}

In general, lifting instruments from Mizuho Medical Co., Ltd (Japan)developed for gasless laparoscopic surgery are used. Among these, instruments that are necessary for gynecological gasless laparoscopic surgery include a lifting bar, lifting handle, Kirschner wire of $1.2 \mathrm{~mm}$ in diameter, pliers and 9Fr Nelaton catheter.

\section{Lap Protector:}

Previously, the abdominal aperture was covered with the peritoneum that was sewed up several points subcutaneously and it was useful for smooth insertion of the forceps. However, as creation of the peritoneal cover for the abdominal aperture was complicated and it had short comings that caused it to narrow the aperture, we started using the Lap Protector' (Hakko Co., Ltd., Nagano, Japan) in 2003. There are many models of Lap Protector of various sizes. Initially, we used the ultra mini model (Hakko FF0504U), which has the smallest ring diameter. However, we currently use the ultra mini model of Tokyo Medical University (Figure 2), which has a shorter upperlower flexible ring distance and is better for all surgeries. Because the original ultra mini model had a long upper-lower flexible ring distance, the Lap Protector did not fit the abdominal aperture except in patients with a very thick abdominal wall. This led to looseness that made the abdominal aperture smaller, thereby causing problems such as when inserting the forceps. When the Tokyo Medical University model is inserted into the abdominal aperture, the aperture is stretched by its tensile force, which protects it while the pressure also offers a hemostatic effect. This enables the abdominal aperture to be used to the fullest.

Trocar: The abdominal port for endoscopy is created through the umbilical fossa (using the fold near the bottom of the umbilicus) by puncturing with a $5 \mathrm{~mm}$ trocar. No sutures are placed after surgery.

Endoscope: Previously, a $10 \mathrm{~mm}$ endoscope was used because

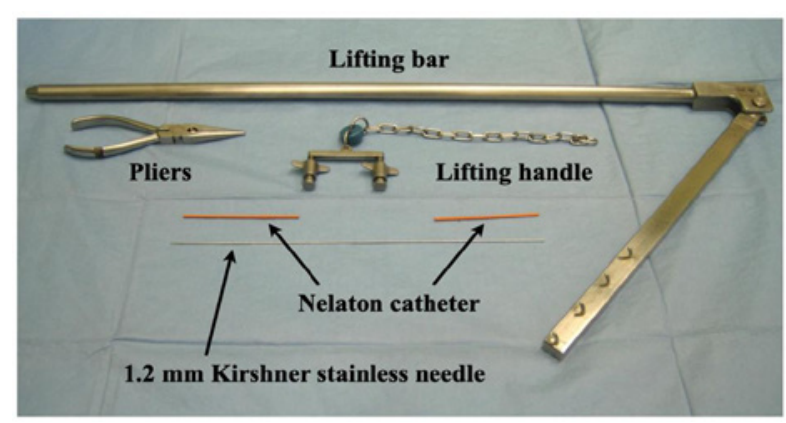

Figure 1: The lifting instruments (a lifting bar, a lifting handle, a $1.2 \mathrm{~mm}$ Kirshner stainless needle, a Nelaton catheter and a pliers) necessary for the subcutaneous abdominal wall method (SAWL).

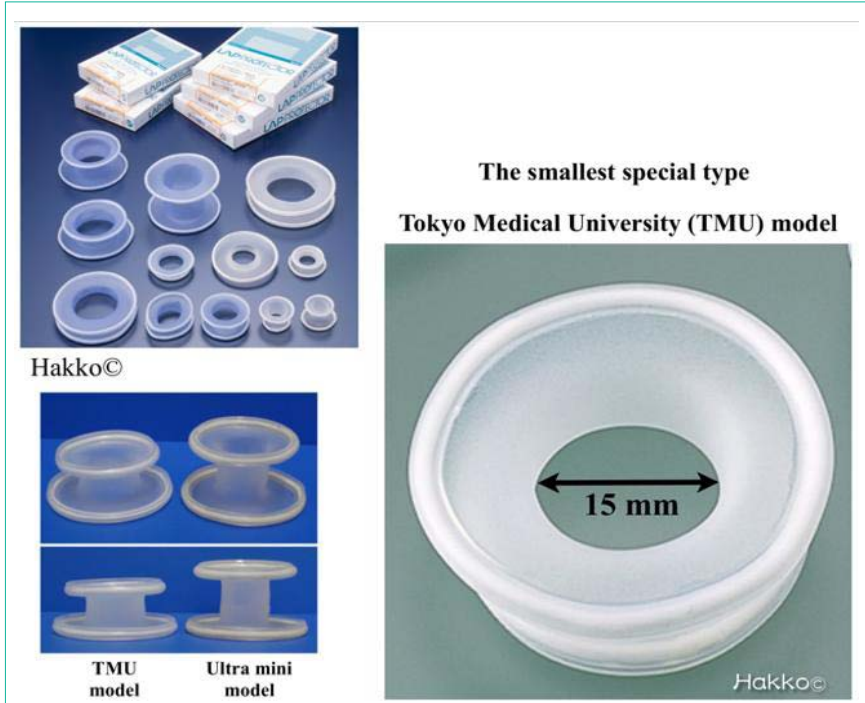

Figure 2: Kinds of Lap Protector ${ }^{\circledR}$

The Tokyo Medical University (TMU) model was made to use it in the abdominal wall aperture of 1.5 to $2.5 \mathrm{~cm}$ diameter.
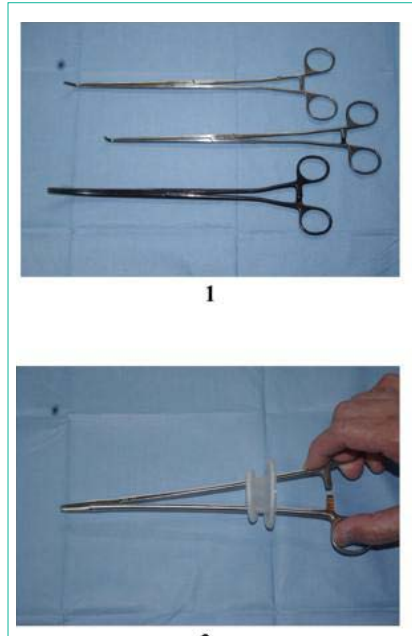


Figure 3: The specialized surgical instruments for SAWL.

1. Long and slim type forceps.

2. The ligators for 1-0, 2-0 and 3-0 threads.

3. The conventional needle holder.

4. The newly developed needle holder with 2 joints.

The use of a conventional needle holder is difficult in a deep cavity because a small abdominal wall aperture restricts its tip from opening. However, the 2 joints on this new needle holder makes it possible for the tip to open enough in a deep cavity.

the first approach was performed using the open method through the umbilicus. However, since the first approach was changed to the open method through the lateral region in 2005, allowing for safe endoscopy-guided trocar puncture, we now use a $5 \mathrm{~mm}$ integratedtype fine endoscope (Olympus video laparoscope). This endoscope has satisfactory resolution comparable to that of the $10 \mathrm{~mm}$ endoscope used in the past, while also offering good maneuverability due to its integrated design.

Surgical instruments: When performing gasless surgery using SAWL, the same surgical instruments used for laparotomy or 


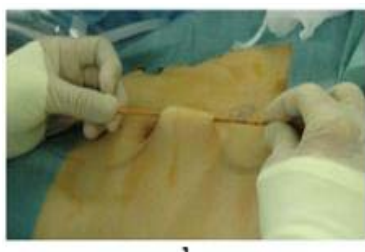

1

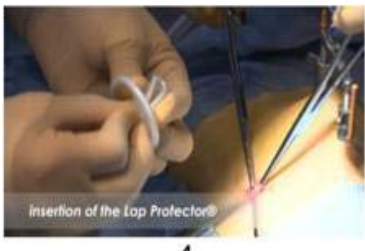

4

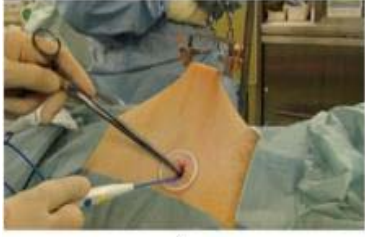

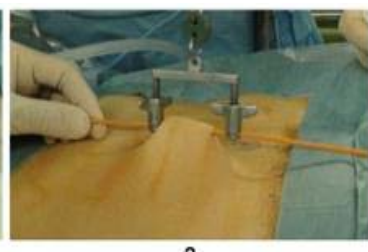

2
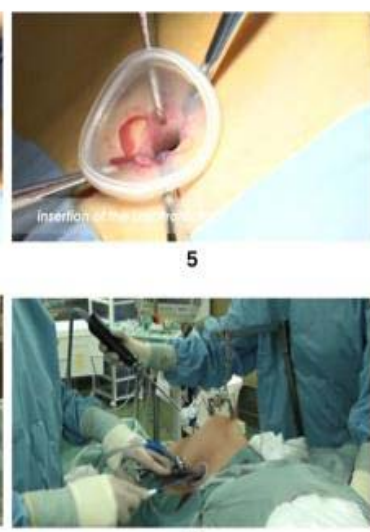
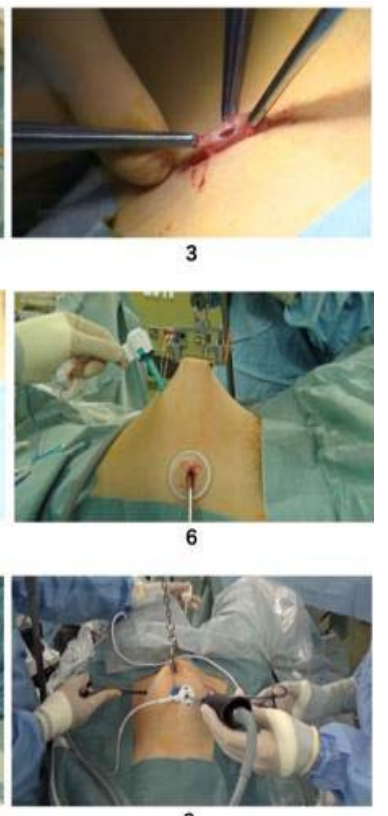

Figure 4: The outside view of procedures for SAWL.

1. A Kirschner wire is inserted subcutaneously on the sagittal line of the median abdominal wall from the suprapubic region towards the umbilicus and passed through a protectiveNelaton catheter

2. A Kirschner wire protected with a Nelaton catheter is fixed to the lifting handle.

3. The freed peritoneum is held with Pean forceps at three sites.

4. Lap protector ${ }^{\circledR}$ is shaped for easy insertion into the abdominal wall aperture.

5. Outside view of SAWL that set up the Lap Protector ${ }^{\circledR}$.

6. A $5 \mathrm{~mm}$ trocar is inserted via the umbilical fossa under surveillance of the endoscope.

7. Two instruments are inserted together into the Lap Protector ${ }^{\circledR}$ to perform the surgery.

8. Outside view of the single-operated port surgery with SAWL.

9. Outside view of the two-operated port surgery with SAWL.

laparoscopic surgery with the pneumoperitoneum method can be used without any problem. However, we use specialized surgical instruments for the galess method that are easier to handle, and that make full use of the characteristics of the abdominal wall lifting method (Figure 3-1, 3-2). We can use a conventional needle holder (Figure 3-3) for suture and ligation, butthe opening of its tip is difficult to control in the deep cavity. Therefore, we developed the needle holder with 2 joints (Figure 3-4) that enabled the opening of its tip in a deep cavity.

Uterine manipulator: Depending on the patient, manipulators such as Uterine Manipulator, RUMI II', or VCare are used.

Other peripheral equipment: Peripheral equipment other than the pneumoperitoneum device basically includes a CCD camera, light source, TV monitor, and recording device as per the pneumoperitoneum method.

\section{Anesthesia}

General anesthesia is usually performed. However, anesthetics that promote intestinal distension such as nitrous oxide are not used. For pregnant patients with ovarian tumors, combined spinal and epidural anesthesia are primarily performed.

\section{Basic technique for SAWL}

In the introduction of gasless laparoscopic surgery with SAWL, the port at the umbilical region for the endoscope was firstly made

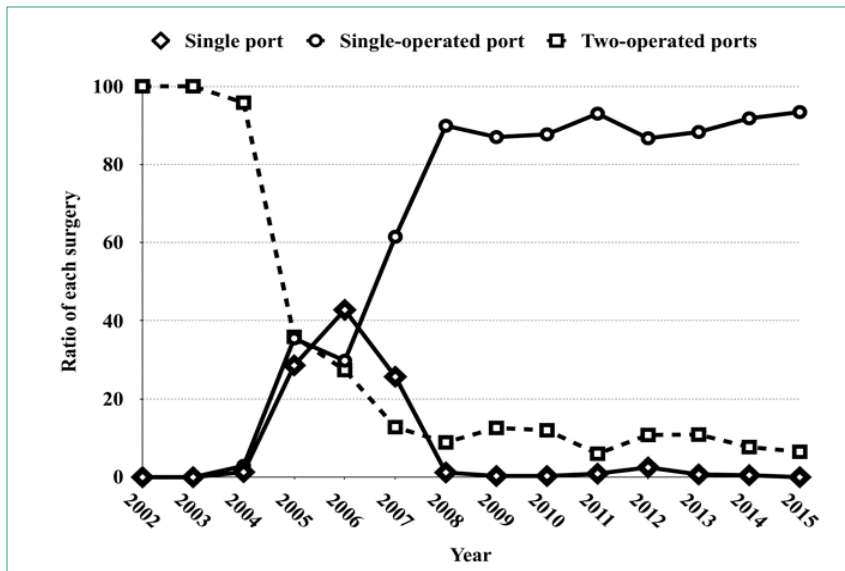

Figure 5: Changes of ratio of each surgery except for Total Laparoscopic Hysterectomy (TLH).

Single-operated port laparoscopic surgery increased year by year since 2005 , and by 2008 it had accounted for almost $90 \%$ of laparoscopic surgeries overallexcept for Total Laparoscopic Hysterectomy (TLH). On the other hand, two-operated ports surgery started to decrease from 2005 and had a usage ratio of approximately $10 \%$.

by open procedure, and then the operated ports at the bilateral lower abdomen were made by open procedure after lifting the abdominal wall. However, we do not use this technique as we now perform the new single-operated port surgery that was developed in 2005 after 


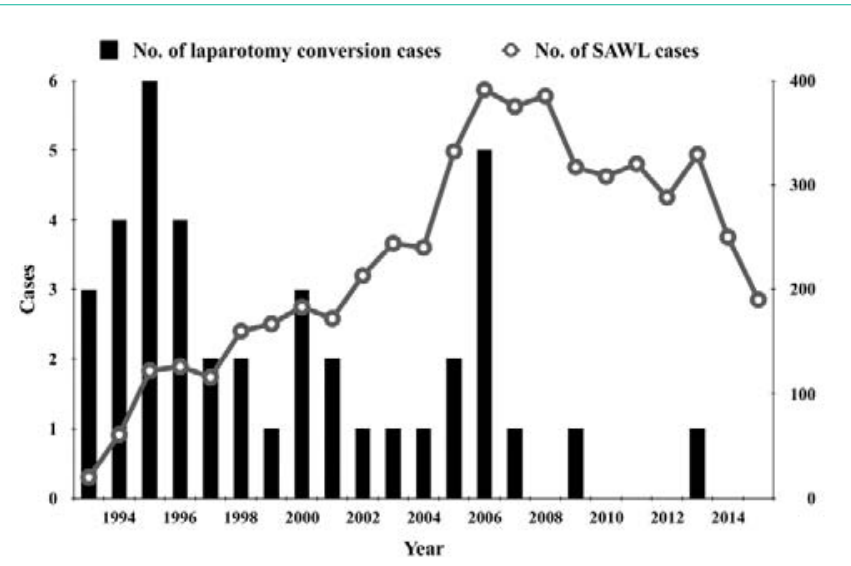

Figure 6: Number of SAWL cases and laparotomy conversion cases. The round circles indicate the number of SAWL cases.

The black bars indicate the number of cases converted to laparotomy.

putting improvement on this method. We demonstrate the details of our currently-used technique as follows.

\section{Abdominal wall lifting:}

- First, a very small incision is made into the skin of the umbilical fossa using a scalpel to facilitate puncture with a $5 \mathrm{~mm}$ trocar.

- The operator and the assistant lift the abdominal wall from the left and right before a Kirschner wire is inserted subcutaneously on the sagittal line of the median abdominal wall from the suprapubic region towards the umbilicus (Figure 4-1). To prevent skin damage by the wire, the wire is passed through a protective Nelatoncatheter, and fixed to the lifting handle (Figure 4-2).

- The bilateral stump portion of the wire is cut $2 \mathrm{~cm}$ laterally, then bent upwards by forceps or a hand. As a support, the abdominal wall is lifted up and fixated to the lifting arm via a chain ring.

Abdominal port formation technique: An abdominal port is formed by making a small incision of approximately $1.5 \mathrm{~cm}$ by scalpel in the lateral wall of the lower abdomen on either the right or left side with the abdominal wall lifted. Then, the fascia underneath is lifted up using Kocher's forceps and an incision is made with scissors. The aponeurosis of external abdominal oblique muscle is bluntly dissected along the muscle layer using long Pean forceps before dissecting and opening out the internal abdominal oblique muscle underneath in the same manner. Then, the abdominal fascia (transverse fascia) is punctured to reach the peritoneum. Next, two hooks are inserted via the incisional wound, the fascia is exposed, the peritoneum is checked, and held using long Pean forceps. Once familiar with this operation, it enables the peritoneum to be blindly held with ease. After holding the peritoneum with Pean forceps in two sites, the operator penetrates the peritoneum to check that there are no intestines contained, and then makes an additional small incision with a scalpel to reach inside the abdominal cavity. The freed peritoneum is held with Pean forceps at three to four sites (Figure 4-3), and a Lap Protector is inserted (Figure 44, 4-5). A $5 \mathrm{~mm}$ endoscope is inserted through the Lap Protector, and the umbilical fossa is punctured with a $5 \mathrm{~mm}$ laparoscopic trocar under direct vision (Figure 4-6). In single-port surgery, a few instruments are inserted together into the Lap Protector to perform

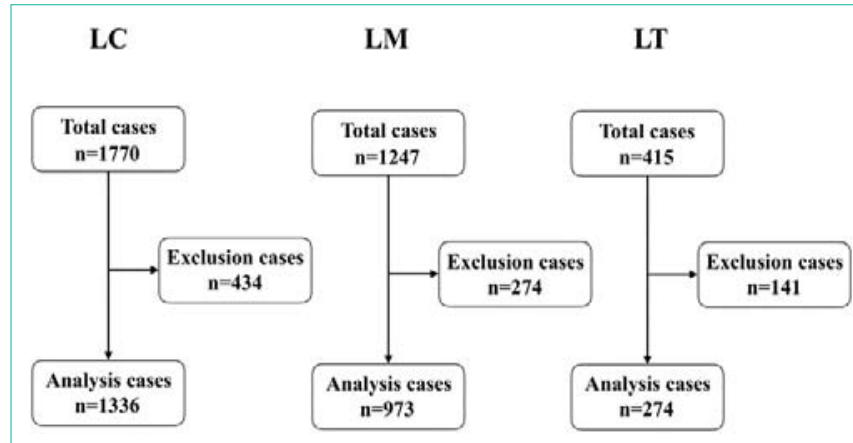

Figure 7: Laparoscopic Cystectomy (LC), Laparoscopic Myomectomy (LM) and Laparoscopic Tubectomy (LT) result overview including inclusion and exclusion data.The inclusion data was gathered from the laparoscopic data record that was made after operation. Cases that had data entry of combined surgery or noentry of the age, BMI, disease name, operative time or estimated blood loss in the record were excluded as the exclusion data.

the surgery (Figure 4-7, 4-8). Furthermore, when two abdominal ports are required for the procedure, a second port is made on the contralateral side in the same manner under direct endoscopic observation (Figure 4-9). As the laparoscopic trocar puncture via the umbilical fossa does not require postoperative wound suturing, therefore offering excellent cosmetic outcomes, we used the fold near the bottom of the umbilicus.

\section{Examination items}

Examination items included age, Body Mass Index (BMI), patient background such as history of laparotomy, as well as the number of annual cases, operation time, estimated blood loss, number of abdominal ports, blood transfusion, and shift to laparotomy.

With regards to Laparoscopic Cystectomy (LC), Laparoscopic Myomectomy (LM), and laparoscopic tubectomy (LT), for which there were many cases, we divided their cases into two stages for the First Stage (FS) we performed surgery with double-operated ports and 10mm endoscope (1993-2005), and during the Second Stage (SS) after the introduction of a single-operated port surgery (2008-2015) for comparison. For statistical analysis, each case data was gathered from the laparoscopic data record that was made after operation as an inclusion data. The cases had data entry of combined surgery or no entry of the age, BMI, disease name, operative time or estimated blood loss in these data records were excluded as exclusion data.

\section{Statistical analysis}

Statistical analysis was carried out using Stata 13 for Windows (StataCorp. LLC, College Station, TX, USA). Outcome variables of operative procedures, operative time and estimated blood loss were measured and described as mean \pm standard deviation in each procedure. A student's t-test was used to compare the outcome variable between the two stages. A multiple linear regression analysis was performed for both outcome variables to control confounding factors such as age and BMI. The statistical significance was considered at $\mathrm{P}<0.05$.

\section{Results}

\section{Number of cases overall and number of cases by surgical procedure}

Since gasless surgery using SAWL was first performed in 1993, 
Table 1: Operative procedures and No. of cases of laparoscopic surgery with SAWL in Tokyo Medical University Hospital (1993-2015)

\begin{tabular}{|c|c|c|}
\hline Operative Procedure & No. of Cases & Rate (\%) \\
\hline LC & 2098 & 39.5 \\
\hline LM & 1738 & 32.7 \\
\hline LT & 510 & 9.6 \\
\hline LA & 369 & 7 \\
\hline TLH & 281 & 5.3 \\
\hline LAVH & 194 & 3.7 \\
\hline Others & 119 & 2.2 \\
\hline Total & 5309 & 100 \\
\hline
\end{tabular}

LC: Laparoscopic Cystectomy; LM: Laparoscopic Myomectomy; LT: Laparoscopic Tubectomy; LA: Laparoscopic Adnexectomy; TLH: Total Laparoscopic Hysterectomy; LAVH: Laparoscopic Assisted Vaginal Hysterectomy.

the number of cases has increased yearly, with the procedure being performed on 391 cases in 2006. Thereafter, from approximately 2012, a decrease in the number of cases was observed. This was attributed to the introduction of robotic surgery in 2009 and the increase in laparoscopic surgery using the pneumoperitoneum method.

By December 2015, we had performed gasless laparoscopic surgery in a total of 5309 patients at our hospital. With regards to the surgical procedure, overall, LC for ovarian tumor was the most frequently performed (2098 patients), followed by LM (1738 patients) and LT (510 patients) (Table 1). Due to the simplicity of the procedure, LC was frequently performed directly after the introduction of gasless surgery. However, LM is currently the most commonly performed procedure. With regards to hysterectomy, after the introduction of Total Laparoscopic Hysterectomy (TLH) in 2005, Laparoscopic Assisted Vaginal Hysterectomy (LAVH) came to be rather rarely performed.

\section{Patient background and surgical findings}

The mean age of patients was 38.0 years, and mean BMI was 21.1 and the ratio of BMI more than 25.0 was $8.2 \% .12 .0 \%$ of patients had a history of laparotomy.

Single-operated port laparoscopic surgery was introduced in 2005 , and by 2008 it had accounted for almost $90 \%$ of laparoscopic surgeries overall except for TLH (Figure 5). On the other hand, double-operated ports surgery started to decrease from 2005 and kept a usage ratio of approximately $10 \%$.

Blood transfusion was required in few cases, with blood transfusion performed in just1 LC patient (FS) (0.1\%), 2 LT patients
(FS: 1 patient, SS: 1 patient; a large amount of intra-peritoneal blood loss was noted preoperatively for both cases) (0.3\%), 2 LM patients (FS: 1 patient, SS: 1 patient $(0.11 \%)$, and 2 TLH patients $(0.3 \%)$.

\section{Shift to laparotomy}

Figure 6 shows the number of laparoscopic surgery patients who underwent gasless surgery using SAWL and the number of patients for whom surgery was shifted to laparotomy according to the years. After 2008 , the procedure was shifted to laparotomy in just 2 patients. Upon examining the reason for shifting to laparotomy, the cause for shifting the procedure to laparotomy was most commonly determined after observation in 26 patients, followed by intraoperative complications, and malignant tumors. The overall rate of shift to laparotomy was 47 out of 5309 patients $(0.89 \%)$. In 11 patients, difficulties in continuing the surgical procedure or postoperative massive bleeding led to laparotomy, with a rate of shift to laparotomy of $0.21 \%$. Incidentally, from 2008, in 2 out of 2688 patients the procedure was shifted to laparotomy, indicating a low rate of shift to laparotomy of $0.07 \%$.

\section{Statistical analysis}

The total numbers of patients who met inclusion criteria was 1770 patients with LC, 1247 patients with LM, and 415 patients with LT. After removing patients who met exclusion criteria, 1336 patients with LC, 973 patients with LM, and 274 patients with LT were in the final analysis (Figure 7). The Table 2 presents the characteristics of the target population by stage and the comparison between FS and SS in each operative procedure. The mean operation time of LC was significantly shorter in SS compared with FS $(\mathrm{P}<0.000)$. A statistically significant difference in mean operation time was also observed in LT patients.

Multivariate linear regression analysis showed that the operation stage was significantly associated with operation time after adjusting for potential confounders but had no significant association with estimated blood loss for LC patients; no significant association was shown between operation stage and operation time although operation stage was significantly associated with estimated blood loss for LM patients; and operation stage was significantly associated with both operation time and estimated blood loss for LT patients (Table 3). Table 4 shows the summary of the statistical analysis.

\section{Discussion}

Since laparoscopic surgery has been fully introduced to cholecystectomy, the advantages of minimally invasive surgery such as shorter hospital stay and earlier return to work are well-matched to the demands of modern society. Thus, laparoscopic surgery has

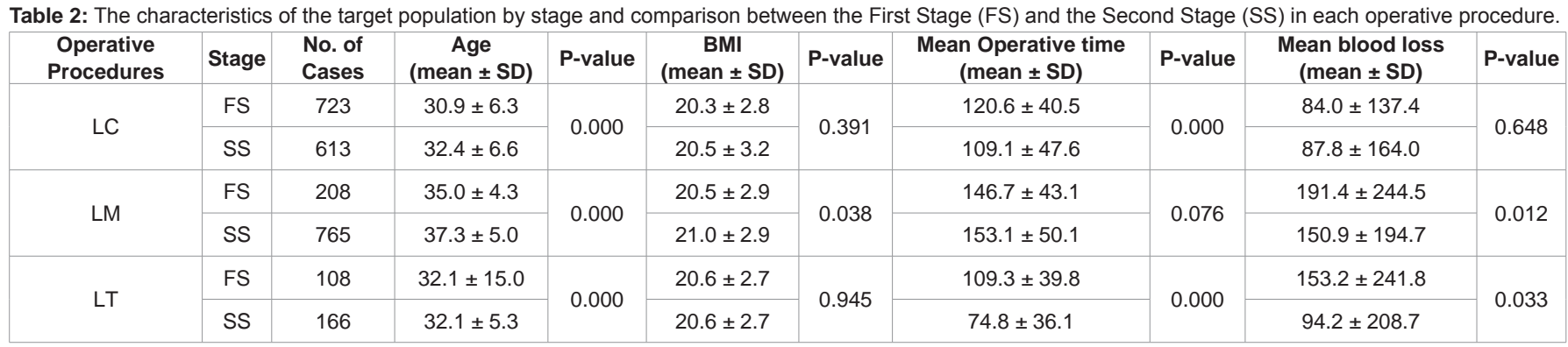

FS: First Stage (1993-2005).

SS: Second Stage (2008-2015). 
Table 3: A linear shape multiple regression analysis for both outcome variables.

\begin{tabular}{|c|c|c|c|c|c|c|c|c|c|c|c|}
\hline \multirow{3}{*}{$\begin{array}{l}\text { Operative } \\
\text { Procedure }\end{array}$} & \multirow{3}{*}{$\begin{array}{c}\text { Factors } \\
\text { SS }\end{array}$} & \multicolumn{5}{|c|}{ Operative Time } & \multicolumn{5}{|c|}{ Estimated Blood Loss } \\
\hline & & \multirow{2}{*}{$\begin{array}{c}\text { Regression } \\
\text { Coefficient } \\
-12.439\end{array}$} & \multirow{2}{*}{$\begin{array}{c}\begin{array}{c}\text { Standard } \\
\text { error }\end{array} \\
2.400\end{array}$} & \multirow{2}{*}{$\begin{array}{c}\text { p-value } \\
0.000\end{array}$} & \multicolumn{2}{|c|}{$\begin{array}{c}95 \% \text { Confidence } \\
\text { interval }\end{array}$} & \multirow{2}{*}{$\begin{array}{c}\begin{array}{c}\text { Regression } \\
\text { Coefficient }\end{array} \\
1.600\end{array}$} & \multirow{2}{*}{$\begin{array}{c}\begin{array}{c}\text { Standard } \\
\text { error }\end{array} \\
8.285 \\
\end{array}$} & \multirow{2}{*}{$\begin{array}{c}\text { p-value } \\
0.847\end{array}$} & \multicolumn{2}{|c|}{$\begin{array}{c}95 \% \text { Confidence } \\
\text { interval }\end{array}$} \\
\hline & & & & & -17.160 & -7.720 & & & & -14.653 & 17.853 \\
\hline \multirow{3}{*}{ LC } & Age & 0.407 & 0.186 & 0.029 & 0.042 & 0.771 & 1.156 & 0.640 & 0.071 & -0.099 & 2.412 \\
\hline & BMI & 1.885 & 0.405 & 0.000 & 1.091 & 2.679 & 2.757 & 1.394 & 0.048 & 0.023 & 5.492 \\
\hline & $\begin{array}{c}\text { Constant } \\
\text { term }\end{array}$ & 69.723 & 9.765 & 0.000 & 50.567 & 88.880 & -7.778 & 33.645 & 0.817 & -73.782 & 58.226 \\
\hline \multirow{4}{*}{ LM } & SS & 3.643 & 3.840 & 0.343 & -3.891 & 11.178 & -46.971 & 16.312 & 0.004 & -78.982 & -14.961 \\
\hline & Age & 0.984 & 0.319 & 0.002 & 0.358 & 1.610 & 0.875 & 1.355 & 0.519 & -1.785 & 3.535 \\
\hline & BMI & 1.917 & 0.537 & 0.000 & 0.863 & 2.971 & 9.545 & 2.282 & 0.000 & 5.065 & 14.024 \\
\hline & $\begin{array}{c}\text { Constant } \\
\text { term }\end{array}$ & 72.844 & 15.465 & 0.000 & 42.495 & 103.194 & -35.337 & 65.703 & 0.591 & -164.270 & 93.600 \\
\hline \multirow{3}{*}{ LT } & SS & -34.509 & 4595 & 0.000 & -43.556 & -25.461 & -58.914 & 27.557 & 0.033 & -113.170 & -4.659 \\
\hline & BMI & 2.433 & 0.838 & 0.004 & 0.783 & 4.082 & 0.185 & 50.240 & 0.971 & -9.706 & 10.075 \\
\hline & $\begin{array}{c}\text { Constant } \\
\text { term }\end{array}$ & 63.616 & 18.162 & 0.001 & 27.858 & 99.373 & 177.435 & 108.917 & 0.104 & 108.917 & 0.104 \\
\hline
\end{tabular}

Table 4: Summary of the statistical analysis.

\begin{tabular}{|c|c|c|c|c|}
\hline \multirow{2}{*}{ Operative Procedure } & \multicolumn{2}{|c|}{ Operative Time } & \multicolumn{2}{|c|}{ Estimated Blood Loss } \\
\hline & Tendency & Significant difference & Tendency & Significant difference \\
\hline LC & Shortening & $\star * *$ & Increase & NS \\
\hline LM & Extension & NS & Decrease & $* * *$ \\
\hline LT & Shortening & $\star \star \star *$ & Decrease & ** \\
\hline
\end{tabular}

rapidly come into widespread use. It was no exception in gynecology, and because laparoscopic surgery is excellent in terms of aesthetic outcomes, it has been widely accepted as the general surgical procedure. However, laparoscopic surgery is also associated with many issues. The greatest problem is caused by pneumoperitoneum, an indispensable method used to ensure the operative field. The pneumoperitoneum procedure of insufflation of the abdominal cavity can lead to various problems such as risks associated with blindly performing the first puncture, the effect of gas absorption and gas pressure on the body, the high level of skill required for surgery in a limited closed space, and the use of high-cost disposable products. While some of these problems have been improved, many remain issues.

Gasless surgery using SAWL was designed to improve these problems. At our department, we first introduced the SAWL to laparoscopic surgery in the field of obstetrics and gynecology in 1993, since which we have consistently endeavored to develop and popularize this procedure through trial and error [6,7]. We have made various improvements to this method that resulted in the currently-used technique (single-operated port surgery). These include immobilizing the site of steel wire insertion, using a uterine manipulator, discontinuing the use of a trocar and sheath for treatment through the abdominal port, using a Lap Protector, creating an abdominal port using the open method, changing the site of abdominal port formation, and reducing the number of abdominal ports (development of the two-ports and single-port laparoscopic surgery [8] with abdominal wall lifting procedure).

In contrast to earlier methods, the single-operated port surgery method introduced in 2005 requires the operator to manipulate all of the forceps, meaning that they must be familiar with manipulating two forceps inserted through a single operated port. However, comparison of the FS and SS revealed that the operation time of SS was significantly shortened or equal, and estimated blood loss of SS was significantly decreased or equal. We believe that this may be attributed to the fact that once the operator has become familiar with manipulating two forceps, they can freely manipulate the forceps better than when manipulating the forceps in cooperation with the assistant. In fact, among LC, LM, and LT patients, the procedure was almost never shifted from single-operated port surgery to twooperated port surgery. Furthermore, with regards to LM, there are advantages that do not exist with the pneumoperitoneum method such as strong traction by single prong tenaculum forceps, morcellation of the uterine fibroids by the scalpel, and easier suture ligation, and therefore this method is considered a superior surgical technique in terms of safety, operability, and cost effectiveness.

The rate of conversion to laparotomy was slightly high, at $0.89 \%$ overall. Detailed examination revealed that the procedure was most commonly shifted to laparotomy after observation. Most instances involved patients with severe endometriosis and interstitial pregnancy early in the FS, at which time it was deemed difficult to perform laparoscopic surgery.

Meanwhile, the rate of conversion to laparotomy due to difficulties continuing surgery intraoperatively and postoperative bleeding was just $0.21 \%$. Furthermore, after the establishment of the present method, the rate of conversion to laparotomy was greatly reduced to $0.07 \%$. This conversion rate is a value achieved by most operators, 
therefore indicating that the present method is deemed a procedure that is rarely shifted to laparotomy.

Gasless laparoscopic surgery with SAWL offers excellent safety and cost efficiency, as well as forceps manipulability such as for suture ligation $[1,2,6,7]$. Therefore, it makes an excellent method for both patients and operators alike. On the basis of our experience, we have found that it can be adapted for most gynecological diseases. However, in patients with a thick abdominal wall, it can be difficult to obtain a good operative field. Based on our data, in patients with a BMI $\geq 35$, we believe that it might be difficult to perform surgery using this method alone. However, when using the pneumoperitoneum method alone, a good operative field often cannot be obtained unless the abdominal air pressure is increased. In such patients, the pneumoperitoneum method combined with abdominal wall lifting can help control intraperitoneal pressure caused by pneumoperitoneum. In the future, we hope to establish safer laparoscopic surgery for severely obese patients by appropriately setting conditions such as appropriate Trendelenburg positions, pneumoperitoneum pressure, and the number and placement of abdominal ports, when combining the pneumoperitoneum method combined with abdominal wall lifting.

\section{References}

1. Nagai H, Inaba T, Kamiya S. A new method of laparoscopic cholecystectomy. An abdominal wall lifting technique without pneumoperitoneum. Abstract Surg laparosc Endosc. 1991; 1: 126.
2. Hashimoto D, Nayeem SA, Kajiwara S, Hoshino T. Laparoscopic cholecystectomy: an approach without pneumoperitoneum. Sur Endosc. 1993; 7: 54-56.

3. Gazayerli MM. The Gazayerli endoscopic retractor model 1. Surg LaparoscEndosc. 1991; 1: 98-100.

4. Kitano S, Iso $\mathrm{Y}$, Tomikawa $\mathrm{M}$, Moriyama $\mathrm{M}$, Sugimachi $\mathrm{K}$. A prospective randomized trial comparing pneumoperitoneum and U-shaped retractor elevation for laparoscopic cholecystectomy. Surg Endosc. 1993; 7: 311-314.

5. Newman L $3^{\text {rd }}$, Luke JP, Ruben DM, Eubanks S. Laparoscopic herniorrhaphy without pneumoperitoneum. Surg LaparoscEndosc.1993; 3: 213-215.

6. Nakajima A, Isaka K, Ogawa T, Kosugi Y, Koshiishi M, Takada J, et al. Gynecological laparoscopic operation by "hanger lifting method". Nihon SankaFujinka Gakkai Zasshi. 1994; 46: 919-920.

7. Isaka K, Ogawa T, Hosaka M, Nakajima A, Kosugi Y, Takayama M. Usefulness of newly developed subcutaneous one point lifting method in gynecological laparoscopic operation. Nihon SankaFujinka Gakkai Zasshi. 1996; 48: 53-60.

8. Nakayama D, Ito H, Nakachi N, Takahashi C, Osakabe Y, Itokazu O, et al. One hole type laparoscopic operation using subcutaneous abdominal wall lifting method. Japanese J GynecolObstetEndosc. 2005; 21: 82. 